\title{
EXISTENCE OF SOLUTIONS TO THE BETHE ANSATZ EQUATIONS FOR THE 1D HUBBARD MODEL: FINITE LATTICE AND THERMODYNAMIC LIMIT
}

\author{
PEDRO S. GOLDBAUM \\ Department of Physics, Princeton University \\ Jadwin Hall, Princeton, NJ 08544, USA.
}

\begin{abstract}
In this work, we present a proof of the existence of real and ordered solutions to the generalized Bethe Ansatz equations for the one dimensional Hubbard model on a finite lattice, with periodic boundary conditions. The existence of a continuous set of solutions extending from any $U>0$ to $U=\infty$ is also shown. This continuity property, when combined with the proof that the wavefunction obtained with the generalized Bethe Ansatz is normalizable, is relevant to the question of whether or not the solution gives us the ground state of the finite system, as suggested by Lieb and Wu. Lastly, for the absolute ground state at half-filling, we show that the solution converges to a distribution in the thermodynamic limit. This limit distribution satisfies the integral equations that led to the well known solution of the 1D Hubbard model.
\end{abstract}

PACS numbers: 71.10.Fd, 71.10.Hf, 71.30.+h.

\section{INTRODUCTION}

In 1968, Lieb and Wu [LW1] solved the one-dimensional Hubbard model [H] , and showed the absence of Mott transition in the ground state. A new version of this result [LW2, with additional details on the exact solution for the ground state, was recently published, motivated by the increasing importance of the 1D Hubbard model in Condensed Matter Physics.

Despite being one of the most studied models to describe interacting electron systems, many questions concerning the Hubbard model remain as open problems, and the 1D Hubbard model is the only one for which an exact solution was found. Still, many results concerning the Bethe Ansatz to the Hubbard model rely on unproved hypotheses. The purpose of this paper is to answer some of the questions raised in the recent paper by Lieb and $\mathrm{Wu}$, concerning the existence of a solution to the Bethe Ansatz equations for the 1D Hubbard model on a finite lattice, and whether or not this solution gives us the ground state. We will also discuss the solution in the thermodynamic limit.

Consider a system with $N$ electrons on a lattice with $N_{a}$ sites. The one-dimensional Hubbard model is described by the hamiltonian

$$
H=-\sum_{i=1}^{N_{a}} \sum_{\sigma}\left(c_{i+1, \sigma}^{\dagger} c_{i, \sigma}+\text { h.c. }\right)+U \sum_{i=1}^{N_{a}} n_{i \uparrow} n_{i \downarrow},
$$

E-mail: goldbaum@princeton.edu. 
where $c_{i, \sigma}^{\dagger}, c_{i, \sigma}$ are the fermion creation and annihilation operators for an electron with spin $\sigma=\uparrow$ or $\downarrow$, at site $i$, and $n_{i \uparrow}, n_{i \downarrow}$ are the corresponding occupation number operators. We are using here periodic boundary conditions $\left(N_{a}+1 \equiv 1\right)$, so we are actually working on a ring with $N_{a}$ sites. Particle-hole symmetry permits us to restrict to the case $N \leqslant N_{a}$.

Since the hamiltonian commutes with $\sum_{i=1}^{N_{a}} n_{i \uparrow}$ and $\sum_{i=1}^{N_{a}} n_{i \downarrow}$, we can look for energy eigenstate with a fixed number of spins up and down. Let us denote these states by $\left|M, M^{\prime}\right\rangle$, where $M$ is the number of spin-down, and $M^{\prime}$ the number of spin-up electrons. Spin-up and spin-down symmetry allows us to restrict to the case $M \leqslant M^{\prime}$.

We can expand $\mid M, M^{\prime}>$ in a basis of states of localized electrons,

$$
\left|M, M^{\prime}>=\sum_{1 \leqslant x_{i} \leqslant N_{a}} f\left(x_{1}, \ldots, x_{N}\right)\right| x_{1}, \ldots, x_{N}>
$$

where $\mid x_{1}, \ldots, x_{N}>$ denotes the state where the spin-down electrons occupy the sites $x_{1}, \ldots, x_{M}$, and the spin-up electrons are located at $x_{M+1}, \ldots, x_{N}$.

Our states are defined in the region $R=\left\{1 \leqslant x_{i} \leqslant N_{a}, i=1, \ldots, N\right\}$. Given any permutation $Q:\{1, \ldots, N\} \rightarrow\{Q 1, \ldots, Q N\}$, we can define the region

$$
R_{Q}=\left\{1 \leqslant x_{Q 1} \leqslant x_{Q 2} \leqslant \ldots \leqslant x_{Q N} \leqslant N_{a}\right\} \subset R
$$

It is clear that

$$
\cup_{Q} R_{Q}=R
$$

where the union is taken over the $N$ ! possible permutations. We should always keep in mind that the $x_{1}, \ldots, x_{M}$ denote the positions of the spin-down electrons.

The solution of the 1D Hubbard model is based on a generalized version of the BetheAnsatz $\left[\mathrm{Be}\right.$. For a sequence of unequal and ordered numbers $k_{1}<k_{2}<\cdots<k_{N}$, we assume that the energy eigenstates are given by (1.1), where the amplitudes $f\left(x_{1}, \ldots, x_{N}\right)$ are given in the region $R_{Q}$ by

$$
f\left(x_{1}, \ldots, x_{N}\right)=\sum_{P} A_{Q}\{P\} \exp \left[i \sum_{j=1}^{N} k_{P_{j}} x_{j}\right] .
$$

The $(N !)^{2}$ coefficients $A_{Q}\{P\}$ have to be determined in order for the state to be well defined in the boundary of two regions $R_{Q}$ and $R_{Q^{\prime}}$. Also, we should make sure that the resulting state is antisymmetric with respect to exchange of two identical particles, and that it satisfies the periodic boundary conditions.

Those restrictions result in a system of $(N !)^{2}$ linear and homogeneous equations for the coefficients $A_{Q}\{P\}$. Fortunately, these equation were studied in detail by Gaudin [G] and Yang $[Y]$, in the context of a system of fermions with delta interaction. The only difference here is that we are working on a lattice, and not in the continuum, so the $k_{j}$ that appear on the system of equations have to be substituted by $\sin k_{j}$, which does not interfere with the algebraic analysis.

The condition obtained in $[\mathrm{G}]$ and $[\mathrm{Y}]$ for the existence of a non-trivial solution for the coefficients $A_{Q}\{P\}$ can be written as the generalized Bethe-Ansatz equations

$$
\begin{aligned}
& e^{i k_{j} N_{a}}=\prod_{\beta=1}^{M} \frac{i \sin k_{j}-i \Lambda_{\beta}-U / 4}{i \sin k_{j}-i \Lambda_{\beta}+U / 4} \quad, \quad j=1, \ldots, N, \\
& \prod_{j=1}^{N} \frac{i \sin k_{j}-i \Lambda_{\alpha}-U / 4}{i \sin k_{j}-i \Lambda_{\alpha}+U / 4}=-\prod_{\beta=1}^{M} \frac{-i \Lambda_{\beta}+i \Lambda_{\alpha}+U / 2}{-i \Lambda_{\beta}+i \Lambda_{\alpha}-U / 2} \quad, \quad \alpha=1, \ldots, M,
\end{aligned}
$$


in terms of our original set $\left\{k_{j}\right\}_{j=1}^{N}$, and a set of auxiliary parameters $\left\{\Lambda_{\alpha}\right\}_{\alpha=1}^{M}$.

Defining

$$
\theta(x)=-2 \tan ^{-1}(2 x / U)
$$

and taking the logarithm of the equations above, we obtain the Lieb-Wu equations

$$
\begin{array}{r}
N_{a} k_{j}=2 \pi I_{j}+\sum_{\beta} \theta\left(2 \sin k_{j}-2 \Lambda_{\beta}\right), \quad j=1, \ldots, N, \\
\sum_{j} \theta\left(2 \sin k_{j}-2 \Lambda_{\alpha}\right)=2 \pi J_{\alpha}-\sum_{\beta} \theta\left(\Lambda_{\alpha}-\Lambda_{\beta}\right), \quad \alpha=1, \ldots, M .
\end{array}
$$

The coefficients $I_{j}$ are integers if $M$ is even, and half-integers otherwise. Similarly, $J_{\alpha}$ are integers if $N-M=M^{\prime}$ is odd and half-integers otherwise. We will restrict to the case where $M$ is odd, and $N$ is even, since in this case the ground state is unique for every $U$. For the ground state, we can choose the $I_{j}$ and $J_{\alpha}$ that will give us the correct solution in the limit $U \rightarrow \infty$. In this limit, the equations decouple, and we have

$$
I_{j}=j-\frac{N+1}{2} \quad, \quad J_{\alpha}=\alpha-\frac{M+1}{2} .
$$

In the thermodynamic limit, taking $N_{a}, N, M, M^{\prime} \rightarrow \infty$, keeping their ratios fixed, (1.5) and (1.6) become integral equations. The analysis of the resulting equations led to the well known solution of the 1D Hubbard model.

Some questions concerning the finite lattice case remained as open problems. The purpose of this paper is to provide rigorous results to some of those problems. In particular, we are going to show that the equations (1.5), (1.6) do indeed have a solution, and that it is ordered in $j$ and $\alpha\left(k_{j+1}>k_{j}, \Lambda_{\alpha+1}>\Lambda_{\alpha}\right)$. The ordering is important in the derivation of the integral equations in the thermodynamic limit. We also show that there is a continuous curve of solutions extending from $U=\infty$ to any $U>0$. We are ready to state our main result:

Theorem 1.1. The equations (1.5) and (1.6) have at least one real solution satisfying

$$
\begin{gathered}
-\pi \leqslant k_{1}<\cdots<k_{N} \leqslant \pi, \\
\Lambda_{1}<\cdots<\Lambda_{M} .
\end{gathered}
$$

Furthermore, there is a continuous curve of solutions defined by $\gamma(t):[0,1] \rightarrow \mathbb{R}^{N+M+1}$, where

$$
\gamma(t)=\left(k_{1}(t), \ldots, k_{n}(t), \Lambda_{1}(t), \ldots, \Lambda_{M}(t), U(t)\right)
$$

extending from any $U>0$ to $U=\infty$.

We know that the state obtained by the solution to (1.5), (1.6) correspond to the true ground state in the limit $U=\infty$, since we chose the coefficients $I_{j}$ and $J_{\alpha}$ in order to match these states. To prove that the ground state is given by the Bethe Ansatz solution for any positive $U$, we need a few additional facts. Since $M$ and $M^{\prime}$ are odd, the ground state is non-degenerate for any $U$ [LW2], and our result shows that starting with the solution at $U=\infty$, and going along the curve $\gamma(t)$, the state is indeed the ground state, provided $f\left(x_{1}, \ldots, x_{N}\right)$ defined by (1.2) does not vanish for all $\left(x_{1}, \ldots, x_{N}\right)$.

On section 4, we prove that the generalized Bethe Ansatz equations have a solution that is algebraic in $U$. Since the Bethe Ansatz wavefunction is a rational function in the variables $z_{j}=e^{i k_{j}}, \Lambda_{\alpha}$ and $U$ (see $[\mathrm{W}]$ ), its norm will have at most finitely many zeros and poles as a function of $U$. We show that we can redefine our wavefunction at these problematic points, in order to obtain the physical solution for all $U$. 
Once the existence of a solution that corresponds to the ground state of our finite system is shown, our next goal is to determine what happens with the set of $k$-s and $\Lambda$-s in the thermodynamic limit. As we increase the lattice size, a sequence of distributions measuring the density of $k$-s and $\Lambda$-s can be defined. In the thermodynamic limit, we show that a subsequence of these distributions converge to the solution of the integral equations derived by Lieb and Wu.

We are particularly interested in the absolute ground state at half-filling, in which case an exact solution can be found, including an explicit expression for the ground state energy. In this case, we show that the whole sequence converges to the explicit solution obtained in LW1.

\section{EXISTENCE OF A SOLUTION}

The goal in this section is to prove the existence of solutions to the Lieb-Wu equations that are ordered in $j$ and $\alpha\left(k_{j+1}>k_{j}, \Lambda_{\alpha+1}>\Lambda_{\alpha}\right)$. The proof will follow from Brouwer's fixed point theorem, but first we need to define a convenient map to apply the theorem.

2.1. Defining the map. Because of the symmetry of the coefficients $I_{j}$ and $J_{\alpha}$, we can look for symmetric solutions of the form $k_{j}=-k_{N-j+1}$, and $\Lambda_{\alpha}=-\Lambda_{M-\alpha+1}$. Therefore, our set of $k$-s and $\Lambda$-s are totally defined by

$$
\begin{aligned}
& \mathbf{k}=\left\{k_{j}\right\}, \quad j=\frac{N}{2}+1, \ldots, N, \\
& \mathbf{\Lambda}=\left\{\Lambda_{\alpha}\right\}, \quad \alpha=\frac{M+3}{2}, \ldots, M .
\end{aligned}
$$

The other components of $\mathbf{k}$ and $\boldsymbol{\Lambda}$, are obtained by the symmetry condition. In particular, $\Lambda_{(M+1) / 2}=0$.

We can define the map $\phi: \mathbb{R}^{\frac{N}{2}+\frac{M-1}{2}} \rightarrow \mathbb{R}^{\frac{N}{2}+\frac{M-1}{2}}$ by

$$
\phi(\mathbf{k}, \boldsymbol{\Lambda})=\left(\mathbf{k}^{\prime}, \boldsymbol{\Lambda}^{\prime}\right)
$$

where $\boldsymbol{\Lambda}^{\prime}$ is defined by

$$
\sum_{j} \theta\left(2 \sin k_{j}-2 \Lambda_{\alpha}^{\prime}\right)=2 \pi J_{\alpha}-\sum_{\beta} \theta\left(\Lambda_{\alpha}-\Lambda_{\beta}\right),
$$

for $(M+3) / 2 \leqslant \alpha \leqslant M$, and $\mathbf{k}^{\prime}$ by

$$
k_{\frac{N}{2}+1}^{\prime}=\max \left\{0, \frac{1}{N_{a}}\left(2 \pi I_{\frac{N}{2}+1}+\sum_{\beta} \theta\left(2 \sin k_{\frac{N}{2}+1}-2 \Lambda_{\beta}\right)\right)\right\},
$$

whereas for $i>\frac{N}{2}+1$

$$
k_{i}^{\prime}=\max \left\{k_{i-1}^{\prime}, \frac{1}{N_{a}}\left(2 \pi I_{i}+\sum_{\beta} \theta\left(2 \sin k_{i}-2 \Lambda_{\beta}\right)\right)\right\} .
$$

The function $\Lambda^{\prime}(\mathbf{k}, \boldsymbol{\Lambda})$ is well defined since the left side of (2.1) is continuous and strictly increasing in $\Lambda_{\alpha}^{\prime}$. It is also clear that $\mathbf{k}^{\prime}(\mathbf{k}, \boldsymbol{\Lambda})$ is continuous, so the map $\phi(\mathbf{k}, \boldsymbol{\Lambda})=\left(\mathbf{k}^{\prime}, \boldsymbol{\Lambda}^{\prime}\right)$ is continuous.

It is also important to observe that

$$
\Lambda_{\alpha}^{\prime}<C_{N, U},
$$


where $C_{N, U}$ is a constant for each value of $N$ and $U$. Indeed, from (2.1) we have

$$
\sum_{j} \theta\left(2 \sin k_{j}-2 \Lambda_{\alpha}^{\prime}\right) \leqslant \frac{(M-1)}{2} 2 \pi+M \pi \leqslant(N-1) \pi,
$$

which implies

or

$$
\frac{4 \Lambda_{\alpha}^{\prime}}{U}<\tan \left(\frac{\pi}{2}-\frac{\pi}{2 N}\right)+\frac{4}{U}
$$

$$
\Lambda_{\alpha}^{\prime}<\frac{U}{4} \tan \left(\frac{\pi}{2}-\frac{\pi}{2 N}\right)+1 \equiv C_{N, U}
$$

2.2. The fixed point theorem. Now we are ready to state the result that allows us to use Brouwer's fixed point theorem.

Lemma 2.1. If we define the domain $\Omega \subset \mathbb{R}^{\frac{N}{2}+\frac{M-1}{2}}$ by

$$
\begin{aligned}
& 0 \leqslant k_{\frac{N}{2}+1} \leqslant \ldots \leqslant k_{N} \leqslant \pi \\
& 0 \leqslant \Lambda_{\frac{M+3}{2}} \leqslant \ldots \leqslant \Lambda_{M} \leqslant C_{N, U}
\end{aligned}
$$

we have

$$
\phi(\Omega) \subset \Omega .
$$

Proof. By definition,

$$
0 \leqslant k_{\frac{N}{2}+1}^{\prime} \leqslant \cdots \leqslant k_{N}^{\prime}
$$

Also,

$$
k_{N} \leqslant \frac{1}{N_{a}}\left[2 \pi\left(\frac{N-1}{2}\right)\right]<\pi \frac{N}{N_{a}} \leqslant \pi .
$$

Regarding $\Lambda_{\alpha}$, we have

$$
\sum_{j} \theta\left(2 \sin k_{j}-2 \Lambda_{\alpha}^{\prime}\right)>0 \quad \Rightarrow \quad \Lambda_{\alpha}^{\prime}>0
$$

Furthermore, the right side of (2.1) is increasing in $\Lambda_{\alpha}$ and $J_{\alpha+1}>J_{\alpha}$, whereas the left side is increasing in $\Lambda_{\alpha}^{\prime}$. Restricting the map $\phi$ to $\Omega$, this implies

$$
\Lambda_{\alpha+1}^{\prime} \geqslant \Lambda_{\alpha}^{\prime} \text {. }
$$

Combining these results with $\Lambda_{M}^{\prime}<C_{N, U}$ we conclude the proof of the lemma.

Since $\Omega$ is compact and convex (in particular, it has no "holes"), there is a continuous and invertible map

$$
\psi: \Omega \rightarrow D^{\frac{N}{2}+\frac{M-1}{2}},
$$

where $D^{\frac{N}{2}+\frac{M-1}{2}}$ is a disk in $(N+M-1) / 2$ dimensions. The map

$$
\psi \circ \phi \circ \psi^{-1}: D^{\frac{N}{2}+\frac{M-1}{2}} \rightarrow D^{\frac{N}{2}+\frac{M-1}{2}}
$$

is continuous and takes the disk into the disk, and by Brouwer's fixed point theorem, it must have a fixed point. Since $\psi$ is one-to-one, the fixed point of $\psi$ corresponds to a fixed point of $\phi$. 
2.3. The fixed point is a solution to the Bethe Ansatz equations. Since we artificially introduced the maximum in the definiton of $\mathbf{k}^{\prime}$, we need to show that the fixed point $\left(\mathbf{k}^{*}, \boldsymbol{\Lambda}^{*}\right)$, such that

$$
\phi\left(\mathbf{k}^{*}, \boldsymbol{\Lambda}^{*}\right)=\left(\mathbf{k}^{*}, \boldsymbol{\Lambda}^{*}\right),
$$

is indeed a solution to the original equations.

In other words, we need to show that

$$
0<k_{\frac{N}{2}+1}^{*}<\cdots<k_{N}^{*}
$$

Taking $k_{j}=0$, we have

$$
k_{j}^{\prime}=\frac{2 \pi I_{j}}{N_{a}}>0 \quad \Rightarrow \quad k_{\frac{N}{2}+1}^{*}>0 .
$$

That also implies

$$
k_{\frac{N}{2}+1}^{*}=\frac{1}{N_{a}}\left[2 \pi I_{\frac{N}{2}+1}+\sum_{\beta} \theta\left(2 \sin k_{\frac{N}{2}+1}^{*}-2 \Lambda_{\beta}\right)\right] .
$$

Let us consider now

$$
k_{\frac{N}{2}+3}^{*}=\max \left\{k_{\frac{N}{2}+1}^{*}, \frac{1}{N_{a}}\left[2 \pi I_{\frac{N}{2}+3}+\sum_{\beta} \theta\left(2 \sin k_{\frac{N}{2}+3}^{*}-2 \Lambda_{\beta}\right)\right]\right\} .
$$

Assuming that $k_{\frac{N}{2}+3}^{*}=k_{\frac{N}{2}+1}^{*}$, we have

$$
k_{\frac{N}{2}+3}^{*}=\max \left\{k_{\frac{N}{2}+1}^{*}, k_{\frac{N}{2}+1}^{*}+\frac{2 \pi}{N_{a}}\right\}>k_{\frac{N}{2}+1}^{*},
$$

which is a contradiction. Therefore, $k_{\frac{N}{2}+3}^{*}>k_{\frac{N}{2}+1}^{*}$, and proceeding by induction we get

$$
k_{\frac{N}{2}+1}^{*}<k_{\frac{N}{2}+3}^{*}<\cdots<k_{N}^{*} .
$$

We have therefore concluded the proof that the fixed point $\left(\mathbf{k}^{*}, \boldsymbol{\Lambda}^{*}\right)$ satisfies (1.5) and (1.6).

\section{Existence of A CONTINUOUS CURVE OF SOlutions}

We want to analyze now what happens to our solutions as $U$ changes. Our goal is to show that there is a connected set of solutions extending from any $U>0$ to $U=\infty$.

From what we have seen in the last section, we know that on the part of the boundary of $\Omega$ defined by $k_{\frac{N}{2}+1}=0$ or $k_{i+1}=k_{i}$, the vector field defined by

$$
\mathbf{v}_{U}(\mathbf{k}, \boldsymbol{\Lambda})=\phi_{U}(\mathbf{k}, \boldsymbol{\Lambda})-(\mathbf{k}, \boldsymbol{\Lambda})
$$

has a normal component that always points inward. Also, for $\Lambda_{\alpha}=0$ we have $\Lambda_{\alpha}^{\prime}>0$, and $\Lambda_{\alpha}=\Lambda_{\alpha+1}$ implies $\Lambda_{\alpha}^{\prime}<\Lambda_{\alpha+1}^{\prime}$. Therefore, the vector field actually points inward in all the boundary of $\Omega$.

All we need to prove then is that given any family of continuous vector fields

$$
\mathbf{v}_{t}: D^{n} \rightarrow \mathbb{R}^{n} \quad, \quad t \in[0,1],
$$

such that the normal component of $\mathbf{v}_{t}$ points inward on the boundary of the disk $D^{n}$, there is a connected subset of $D^{n} \times[0,1]$ in which $\mathbf{v}_{t}(\mathbf{k}, \boldsymbol{\Lambda})=0$, extending all the way from $t=0$ to $t=1$.

The proof of this statement is trivial if for each $t \in[0,1]$ the vector field $\mathbf{v}$ is nondegenerate. (Here, we consider a vector field to be non-degenerate if, for every point $x$ 
such that $\mathbf{v}(x)=0$, the determinant of the jacobian $\left|\partial_{j} v_{i}\right|$ does not vanish.) In this case, since the zeros of the vector field are always isolated, each zero at $t=0$ will follow a smooth curve as we increase $t$ from 0 to 1 , by the implicit funtion theorem.

In the general case, however, zeros can collide with each other and disappear, as we change $t$, so the proof is not that simple. Let us assume now that we have a general family of vector fields, possibly degenerate.

We can always approximate $\mathbf{v}_{t}$ by a polynomial vector field, in the sense that for every $\epsilon>0$ there is a polynomial vector field $\mathbf{p}$ such that $\left\|\mathbf{v}_{t}-\mathbf{p}\right\|<\epsilon$. If $\mathbf{v}_{t}$ is a polynomial, it will have a finite number of sets of connected zeros. We can define an index for every isolated set of zeros. For an isolated zero $\mathbf{x}_{0}$, we define its index GP] by the degree of the map $\phi: S_{\epsilon} \rightarrow S^{n-1}$ defined by

$$
\phi(\mathbf{x})=\frac{\mathbf{v}_{t}}{\left|\mathbf{v}_{t}\right|},
$$

where $S_{\epsilon}$ is a sphere center at $\mathbf{x}_{0}$ with radius $\epsilon$, and $\epsilon$ is sufficiently small so that $\mathbf{x}_{0}$ is the only zero of $\mathbf{v}_{t}$ in its interior. So the index is just a measure of how many times we map the sphere $S_{\epsilon}$ into the sphere $S^{n-1}$.

For any connected set of zeros, we can define the index analogously, by isolating the set from other zeros by a surface that can be smoothly deformed into a sphere. Alternatively, we could define the index by adding a small perturbation to remove the degeneracy, and adding the index of all resulting isolated zeros. A general definition of the index for vector fields can be found in [GS].

Since $\mathbf{v}_{t}$ always points inwards in the boundary of the disk, the sum of the indices of all connected sets of zeros is equal to one, for all $t$. As $t$ varies from 0 to 1 , the zeros describe a continuous trajectory. A given zero of index +1 cannot simply disappear, unless it collides with a zero of index -1 . Every connected set of zeros can at some point break up into other sets, provided the sum of indices is preserved. Also, new sets of zeros can be created, provided their total index is 0 .

Let $Z \subset D^{n} \times[0,1]$ be the set of all zeros of $\mathbf{v}$, where we now drop the index $t$, and consider the vector field defined on the cylinder $D^{n} \times[0,1]$. If a zero of $\mathbf{v}_{0}$ with index +1 is not connected to a zero of $\mathbf{v}_{1}$ by $\mathrm{Z}$, then it has to be connected to another zero of $\mathbf{v}_{0}$ with index -1 . Since the total index is always equal to 1 , there are not enough zeros of index -1 to annihilate all the +1 zeros. and there will be at least one connected set of zeros from $t=0$ to $t=1$.

We can find a sequence of vector fields $\left\{\mathbf{w}_{n}\right\}$, such that $\mathbf{v}+\mathbf{w}_{n}$ is a polynomial vector field and $\lim _{n \rightarrow \infty}\left|\mathbf{w}_{n}\right|=0$. For each $n$, we have a continuous curve of zeros of $\mathbf{v}+\mathbf{w}_{n}$, from $t=0$ to $t=1$. What we need to do is to prove that the curve cannot be disrupted as we take the limit $\left|\mathbf{w}_{n}\right| \rightarrow 0$.

Let us also define the set

$$
Z^{\prime}=D_{0} \cup Z \cup D_{1}
$$

where $D_{0}$ is the disk at $t=0$, and $D_{1}$ the disk at $t=1$. Consider the topology $\tau^{*}$ in $Z^{\prime}$ defined by taking the intersection of the open sets in the metric topology $\tau$ of $D \times[0,1]$ with $Z^{\prime}$.

Let us assume for a moment that there is no subset of $Z$ connecting $D_{0}$ to $D_{1}$. In this case, we can find two disjoint open sets $X_{0}, X_{1} \in \tau^{*}$ such that

$$
D_{0} \subset X_{0} \quad, \quad D_{1} \subset X_{1},
$$

and $X_{0} \cup X_{1}=Z^{\prime}$. But $X_{0}$ and $X_{1}$ are closed sets in the metric topology $\tau$ (defined by the open balls in the usual metric on the cylinder), since they are given by zeros of a 
continuous vector field. Therefore we can find disjoint open sets $O_{1}, O_{2} \in \tau$ such that

$$
X_{0} \subset O_{0} \quad, \quad X_{1} \subset O_{1} .
$$

But the set $\left(O_{1} \cap O_{2}\right)^{c}$ is nonempty and closed, so

$$
|\mathbf{v}(x)| \neq 0 \quad, \quad \forall x \in\left(O_{1} \cap O_{2}\right)^{c},
$$

implies

$$
|\mathbf{v}(x)|>\delta \quad, \quad \forall x \in\left(O_{1} \cap O_{2}\right)^{c} .
$$

Hence, for $|\mathbf{w}|$ sufficiently small,

$$
|(\mathbf{v}+\mathbf{w})(x)|>\delta / 2,
$$

and there is no curve of zeros of $\mathbf{v}+\mathbf{w}$ connecting $D_{0}$ to $D_{1}$. This is in contradiction with the existence of a sequence of polynomial vector fields converging to $\mathbf{v}$.

Therefore, we conclude the proof of the existence of a connected set of solutions of the Bethe-Ansatz equations, extending from any $U>0$ to arbitrarily large values. Along this curve, the energy given by

$$
E(U(t))=-2 \sum_{j=1}^{N} \cos k_{j}(t)
$$

is continuous in $t$.

\section{NON-VANISHING NORM OF THE WAVEFUNCTION}

As we discussed before, the existence of a continuous curve of solutions implies that the right side of (1.1) is indeed the true ground state, provided that its norm is not zero. However, since we are working on a lattice, and not in the continuum, the wavefunction (1.1) could in principle vanish $\left(f\left(x_{1}, \ldots, x_{N}\right) \equiv 0\right)$, even though the coefficients $A_{Q}\{P\}$ are not all zero. Therefore, to complete the proof that (1.1) is the true ground state, we need to prove that $f\left(x_{1}, \ldots, x_{N}\right)$ does not vanish identically.

We will follow here the same strategy used by C. N. Yang and C. P. Yang [YY] to prove that the Bethe Ansatz does indeed give the true ground state for the anisotropic Heisenberg model.

Let us first define the variables $z_{j}$ by

$$
z_{j}=\left\{\begin{array}{l}
e^{i k_{j}}, j=1, \ldots, N \\
\Lambda_{j-N}, j=N+1, \ldots, N+M .
\end{array}\right.
$$

We can write the equations (1.3), (1.4) as polynomial equations in the variables $z_{j}$ and $U$. So we have a system of $N+M$ polynomial equations, in $N+M+1$ variables,

$$
p_{i}\left(z_{j}, U\right)=0 \quad, \quad i=1, \ldots, N+M,
$$

and our goal is to find solutions given by algebraic functions $z_{j}(U)$.

Let us remind ourselves of some basic facts concerning polynomial equations in several variables. A more detailed background on the subject can be found in [CLO]. Consider the space of polynomials in $n$ complex variables, with complex coefficients, which we denote by $\mathbb{C}\left[x_{1}, \ldots, x_{n}\right]$. Given a set of polynomials $\left\{f_{i}\right\} \subset \mathbb{C}\left[x_{1}, \ldots, x_{n}\right]$, where $i=1, \ldots, m$, we can define an ideal $I\left(f_{1}, \ldots, f_{m}\right) \subset \mathbb{C}\left[x_{1}, \ldots, x_{n}\right]$ by

$$
I\left(f_{1}, \ldots, f_{m}\right)=\left\{\sum_{i=1}^{m} h_{i} f_{i}, h_{1}, \ldots, h_{m} \in \mathbb{C}\left[x_{1}, \ldots, x_{n}\right]\right\} .
$$


We can also define an affine variety $V(I) \subset \mathbb{C}^{n}$ by

$$
V(I)=\left\{\left(a_{1}, \ldots, a_{n}\right) \in \mathbb{C}^{n}: f\left(a_{1}, \ldots, a_{n}\right)=0, \forall f \in I\right\} .
$$

$V(I)$ is the set of solutions to the original polynomial equations

$$
f_{1}\left(x_{1}, \ldots, x_{n}\right)=f_{2}\left(x_{1}, \ldots, x_{n}\right)=\cdots=f_{m}\left(x_{1}, \ldots, x_{n}\right)=0 .
$$

We say that the set of polynomials $\left\{f_{j}\right\}$ form a basis to the ideal $I$. But an ideal can be defined by many bases. A particularly convenient basis for an ideal is the Groebner basis (see appendix A or CLO for details). The Groebner basis is a set of polynomials with the same roots as the original set, with the nice property that it contains polynomials where some of the variables are eliminated. We will be using a Groebner basis for the ideal defined by the Bethe Ansatz equations shortly, but we still need to define the projection of an affine variety.

Given $V(I)$, we can define $\pi^{(k)}: V(I) \subset \mathbb{C}^{n} \rightarrow \mathbb{C}$ by

$$
\pi^{(k)}\left(a_{1}, \ldots, a_{n}\right)=a_{k}
$$

This projection gives us the the values of $x_{k}$ for which the system of polynomial equations has at least one solution.

We are ready to state the technical result that will assist us in our proof.

Lemma 4.1. Let $V \subset \mathbb{C}^{n}$ be an affine variety. Then one of the following has to be true:

- $\pi^{(k)}(V)$ consists of finitely many points (possibly zero),

- $\mathbb{C}-\pi^{(k)}(V)$ consists of finitely many points (possibly zero).

Proof: If we define the ideal $I^{(k)}=I \cap \mathbb{C}\left[x_{k}\right]$, given by the polynomials in $I$ that depend only on the variable $x_{k}$, we have

$$
V\left(I^{(k)}\right)-W \subset \pi^{(k)}(V) \subset V\left(I^{(k)}\right),
$$

where $W$ is a proper subset of $V\left(I^{(k)}\right)$. The right side of this relation is trivial, since $\left(a_{1}, \ldots, a_{k}, \ldots, a_{n}\right) \in V(I)$ implies that $a_{k}$ is a root of any polynomial in $I^{(k)}$. The left side follows from CLO (Theorem 3, p.123).

But $V\left(I^{(k)}\right)$ and $W$ are affine varieties in $\mathbb{C}$, which can either be $\mathbb{C}$ or a set with finitely many points (roots of a polynomial equation $p\left(x_{k}\right)=0$ ). If $V\left(I^{(k)}\right)=\mathbb{C}, W$ is a finite set and $\mathbb{C}-\pi^{(k)}(V)$ consists of finitely many points. Otherwise, $\pi^{(k)}(V)$ is finite.

As a first application of this lemma, we see that our system of polynomial equations will actually have a solution for any complex $U$, except for at most finitely many values, since we proved that it has at least one solution for $U$ real and positive,. If we choose the ordering $z_{1}>z_{2}>\cdots>z_{N+M}>U$, our Groebner basis will not have any polynomial depending only on $U$. Therefore, our last polynomial in the basis should be of the form

$$
p\left(z_{M+N}, U\right)=0 .
$$

There is nothing special about the ordering we chose, so can also construct a Groebner basis with the ordering $z_{1}>\cdots>z_{k}>U$, for any $k$ between 1 and $N+M$. Therefore, the solutions of (4.1) will have to satisfy

$$
\begin{aligned}
p^{(1)}\left(z_{1}, U\right) & =0, \\
p^{(2)}\left(z_{2}, U\right) & =0, \\
\vdots & \\
p^{(M+N)}\left(z_{M+N}, U\right) & =0 .
\end{aligned}
$$


Let us assume for a moment that none of these polynomials vanishes identically. Then, we can factorize each of them into irreducible polynomials

$$
p^{(k)}\left(z_{k}, U\right)=\Pi_{j=1}^{l} p_{l}^{(k)}\left(x_{k}, U\right) .
$$

Every solution will be given by roots of some combination of $p_{l}^{(k)}$. Since there are finitely many combinations, and infinite solutions, there will be one particular combination that will give us solutions for all but finitely many values of $U$ (we are again using Lemma 4.1).

If one of these polynomials vanishes identically, it means that our system of equations is degenerate, and we can add equations to it, reducing the set of solutions, in such a way that the previous argument will work for the extended set of polynomial equations.

Therefore, each of our variables $z_{k}$ will be an algebraic function of $U$ defined by

$$
p_{l}^{(k)}\left(z_{k}, U\right)=0 \text {. }
$$

Since we know that our wavefunction is a rational function in $z_{k}$ and $U$, if we go from $U=\infty$ to any finite $U>0$ along the set of solutions defined by (4.3), our wavefunction will have finitely many zeros and poles. Since those are isolated, we can redefine the wavefunction at these points by the limit of the normalized wavefunctions as we approach them, since the energy of those states varies continuously and is equal to the ground state energy for all $U$. Again, this idea goes back to $[\mathrm{YY}$, in the context of a spin system. That concludes the proof that the Bethe Ansatz gives us the true ground state of the system for any $U>0$.

If $N / N_{a} \leqslant 2 / 3$, we could actually prove a slightly stronger version of this result, in which we show that the wavefunction that results from the solution of (1.5) and (1.6) is not zero for all $U>0$.

Theorem 4.2. If the density of electrons per site satisfies

$$
\frac{N}{N_{a}} \leqslant \frac{2}{3}
$$

the norm of the state given by the Bethe-Ansatz method is strictly positive:

$$
\left\|\sum_{1 \leqslant x_{i} \leqslant N_{a}} f\left(x_{1}, \ldots, x_{N}\right) \mid x_{1}, \ldots, x_{N}>\right\|>0 .
$$

Therefore, the Bethe-Ansatz gives us the true and unique ground state of the system.

Proof: Let us start by considering the case $M=M^{\prime}=N / 2$. Since we know that for a given solution of (1.5) and (1.6), the coefficients $A_{Q}\{P\}$ are not all equal to zero, we just need to show that $f\left(x_{1}, \ldots, x_{N}\right) \equiv 0$ in the region $R$ implies

$$
A_{Q}\{P\}=0,
$$

for any $P$ and $Q$.

Let us consider the region $R_{0}$ defined by

$$
R_{0}=\left\{1 \leqslant x_{1} \leqslant x_{2} \leqslant \ldots \leqslant x_{N} \leqslant N_{a}\right\} \subset R .
$$

Gaudin $\mathrm{G}$ showed that $f\left(x_{1}, \ldots, x_{N}\right)$ in $R$ is totally determined by its value in the region $R_{0}$, and $A_{0}\{P\}=0$, for any $\mathrm{P}$, implies $A_{Q}\{P\}=0$, for all $P, Q$. Therefore we just need to show that $f\left(x_{1}, \ldots, x_{N}\right) \equiv 0$ implies $A_{0}\{P\}=0$, for any P. We will denote the restriction of $f\left(x_{1}, \ldots, x_{N}\right)$ to $R_{0}$ by

$$
f_{0}\left(x_{1}, \ldots, x_{N}\right)=\sum_{P} A_{0}\{P\} \exp \left[i \sum_{j=1}^{N} k_{P_{j}} x_{j}\right] \text {. }
$$


Also in [G], it is shown that for any two permutations $P$ and $Q$, such that $Q$ only acts on variables corresponding to one kind of spin, we have

$$
A_{0}\{P Q\}=I(Q) A_{0}\{P\}
$$

where $I(Q)$ is the sign of the permutation $Q$. This can be proved by combining the boundary conditions at the different regions $R_{Q}$ with the antisimmetry of the wavefunction with respect to exchange of identical particles. In particular, if $P$ and $P^{\prime}$ differ only by the exchange of coordinates of two identical particles,

$$
A_{0}\left\{P^{\prime}\right\}=-A_{0}\{P\}
$$

Therefore, we have

$$
\begin{gathered}
f_{0}\left(x_{2}, x_{1}, \ldots, x_{N}\right)=\sum_{P} A_{0}\{P\} \exp \left[i\left(k_{P 1} x_{2}+k_{P 2} x_{1}+\cdots+k_{P N} x_{N}\right)\right]= \\
-\sum_{P^{\prime}} A_{0}\left\{P^{\prime}\right\} \exp \left[i\left(k_{P^{\prime} 1} x_{1}+k_{P^{\prime} 2} x_{2}+\cdots+k_{P N} x_{N}\right)\right]=-f_{0}\left(x_{1}, x_{2}, \ldots, x_{N}\right) .
\end{gathered}
$$

Notice that this is not a trivial consequence of the antisymmetry alone, since $f_{0}$ is not the true wavefunction when $x_{2}<x_{1}$.

As a result, we have that $f\left(x_{1}, \ldots, x_{N}\right) \equiv 0$ implies $f_{0}\left(x_{1}, \ldots, x_{N}\right)=0$ in the region

$$
\left\{1 \leqslant x_{1}, \ldots, x_{M} \leqslant x_{M+1}, \ldots, x_{N} \leqslant N_{a}\right\} \subset R
$$

If $N_{a} \geqslant 3 N / 2$, that includes

$$
S=\left\{1 \leqslant x_{1}, \ldots, x_{M} \leqslant N, N+1 \leqslant x_{M+1}, \ldots, x_{N} \leqslant 3 N / 2\right\} \subset R
$$

If $f_{0}$ is zero in $S$, we can fix $x_{2}, \ldots, x_{N}$ and consider the system of equations

$$
f_{0}\left(x_{1}, x_{2}, \ldots, x_{N}\right)=0 \quad, \quad 1 \leqslant x_{1} \leqslant N
$$

or

$$
e^{i k_{1} x_{1}} C^{(1)}\left(x_{2}, \ldots, x_{N}\right)+\cdots+e^{i k_{N} x_{1}} C^{(N)}\left(x_{2}, \ldots, x_{N}\right)=0 \quad, \quad 1 \leqslant x_{1} \leqslant N,
$$

where each $C^{(n)}$ is given by a sum over permutations such that $P(1)=n$ :

$$
C^{(n)}=\sum_{P: P 1=n} A_{0}\{P\} \exp \left[i\left(k_{P 2} x_{2}+\cdots+k_{P N} x_{N}\right)\right]
$$

Therefore, (4.4) is a homogeneous system of $N$ linear equations in the variables $C^{(N)}$. The determinant

$$
\left(\begin{array}{cccc}
e^{i k_{1}} & e^{i k_{2}} & \cdots & e^{i k_{N}} \\
e^{2 i k_{1}} & e^{2 i k_{2}} & \cdots & e^{2 i k_{N}} \\
\vdots & \vdots & \ddots & \vdots \\
e^{N i k_{1}} & e^{N i k_{2}} & \cdots & e^{N i k_{N}}
\end{array}\right)=\prod_{j<i}\left(e^{i k_{i}}-e^{i k_{j}}\right) \neq 0
$$

does not vanish, since $-\pi<k_{1}<\cdots<k_{N}<\pi$, and we have

$$
C^{(n)}=0 \quad, \quad 1 \leqslant n \leqslant N
$$

We can fix $x_{3}, \ldots, x_{N}$, and write

$$
C^{(n)}=\sum_{m \neq n} e^{i k_{m} x_{2}} C^{(n, m)}\left(x_{3}, \ldots, x_{N}\right)=0 \quad, \quad 1 \leqslant x_{2} \leqslant N-1,
$$


where

$$
C^{(n, m)}=\sum_{P: P 1=n, P 2=m} A_{0}\{P\} \exp \left[i\left(k_{P 3} x_{3}+\cdots+k_{P N} x_{N}\right)\right] .
$$

Again, the determinant does not vanish and

$$
C^{(n, m)}=0 .
$$

After repeating this argument for all spin-down variables, we will have only $M=N / 2$ pseudo-momenta $k_{i}$ in each term. That is why it suffices to have $N+1 \leqslant x_{M+1}, \ldots, x_{N} \leqslant$ $3 N / 2$. By the time we get to the variable $x_{N}$, we will have

$$
A_{0}\{P\}=0
$$

for all $P$.

If the set $\left\{k_{i}\right\}$ is given by a solution of the equations (1.5) and (1.6), they correspond to a non trivial set of coefficients $A_{0}\{P\}$, and by the result above, $f\left(x_{1}, \ldots, x_{N}\right) \neq 0$ for some $\left(x_{1}, \ldots, x_{N}\right) \in R$, and the state given by the right side of (1.1) has a strictly positive norm. That concludes the proof of the theorem.

In general, $M \neq M^{\prime}$, and the argument above still applies provided $N_{a} \geqslant N+M$ (which includes $N_{a} \geqslant 3 N / 2$ as a particular case).

\section{THE THERMODYNAMIC LIMIT}

Let us now analyze the behavior of the solutions $\left\{k_{j}, \Lambda_{\alpha}\right\}$ as we take the thermodynamic limit, by taking $N_{a} \rightarrow \infty$, keeping the ratios $n=N / N_{a}$ and $m=M / N_{a}$ fixed.

We can define

$$
\rho_{i}(k)=\frac{1}{N_{a}\left|k_{j+1}-k_{j}\right|} \quad, \quad k_{j} \leqslant k<k_{j+1},
$$

and $\rho_{i}(k)=0$ for $k<k_{1}$ or $k \geqslant k_{N}$. The above expression defines a sequence of functions $\rho_{i} \in L^{1}([-\pi, \pi])$. As $i$ increases, so does the number of sites, and $i \rightarrow \infty$ corresponds to $N_{a} \rightarrow \infty$. Analogously, we can define $\sigma_{i}(\Lambda) \in L^{1}(\mathbb{R})$ by

$$
\sigma_{i}(\Lambda)=\frac{1}{N_{a}\left|\Lambda_{\beta+1}-\Lambda_{\beta}\right|} \quad, \quad \Lambda_{\beta} \leqslant \Lambda<\Lambda_{\beta+1}
$$

and $\sigma_{i}(\Lambda)=0$ for $\Lambda<\Lambda_{1}$ or $\Lambda \geqslant \Lambda_{M}$. From the definition, the $L^{1}$ norm of $\rho_{i}$ and $\sigma_{i}$ satisfy

$$
\left\|\rho_{i}\right\|_{L^{1}}=\frac{N-1}{N_{a}}=n-\frac{1}{N_{a}} \quad, \quad\left\|\sigma_{i}\right\|_{L^{1}}=\frac{M-1}{N_{a}}=m-\frac{1}{N_{a}} .
$$

In particular, these functions are uniformly bounded as we take the thermodynamic limit.

Let us also define

$$
f_{i}(k)=N_{a} k-\sum_{\beta} \theta\left(2 \sin k-2 \Lambda_{\beta}\right) .
$$

From the Lieb-Wu equations, we have $f_{i}\left(k_{j}\right)=2 \pi I_{j}$. Also,

$$
f_{i}^{\prime}(k) \leqslant N_{a}+\frac{8}{U} M \leqslant N_{a}\left(1+\frac{8 m}{U}\right),
$$

which implies

or

$$
f_{i}\left(k_{j+1}\right)-f_{i}\left(k_{j}\right)=2 \pi \leqslant\left|k_{j+1}-k_{j}\right| N_{a}\left(1+\frac{8 m}{U}\right),
$$

$$
\rho_{i}(k) \leqslant \frac{(1+8 m / U)}{2 \pi} .
$$


Since $\rho_{i}(k)$ is bounded, $\rho_{i} \in L^{p}([-\pi, \pi])$, for $1 \leqslant p \leqslant \infty$. The sequence $\left\{\rho_{i}\right\}$ is actually uniformly bounded in $L^{p}$.

With a similar goal, we define

$$
g_{i}(\Lambda)=\sum_{j} \theta\left(2 \sin k_{j}-2 \Lambda\right)+\sum_{\beta} \theta\left(\Lambda-\Lambda_{\beta}\right)
$$

such that $g_{i}\left(\Lambda_{\alpha}\right)=2 \pi J_{\alpha}$. Its derivative is bounded by

$$
g_{i}^{\prime}(\Lambda) \leqslant \frac{8}{U} \sum_{j} \frac{1}{1+\left(\frac{4 \sin k_{j}-4 \Lambda}{U}\right)^{2}} \leqslant \frac{8 N_{a} n}{U} .
$$

Once more, that implies

$$
g_{i}\left(\Lambda_{\alpha+1}\right)-g_{i}\left(\Lambda_{\alpha}\right)=2 \pi \leqslant\left|\Lambda_{\alpha+1}-\Lambda_{\alpha}\right| N_{a} \frac{8 n}{U},
$$

or

$$
\sigma_{i}(\Lambda) \leqslant \frac{4 n}{\pi U}
$$

The first inequality on (5.4) shows that all $g_{i}^{\prime}$ are bounded by a function in $L^{2}(\mathbb{R})$. Therefore, $\left\{\sigma_{i}\right\}$ is uniformly bounded in $L^{2}$.

Since both $\left\{\rho_{i}\right\}$ and $\left\{\sigma_{i}\right\}$ are uniformly bounded, we can use Banach-Alaoglu theorem to show that there is a subsequence converging weakly in $L^{2}$ (see [LL]):

$$
\rho_{n_{i}} \rightarrow \rho_{w} \quad, \quad \sigma_{n_{i}} \rightarrow \sigma_{w} .
$$

We will be working now with this subsequence, but we will keep the index as $i$ just to simplify the notation. Our goal is to show that this subsequence also converges pointwise.

If we take equation (1.5) with index $j$, subtract it from the same equation with index $j+1$, and divide the result by $2 \pi N_{a}\left|k_{j+1}-k_{j}\right|$, we obtain

$$
\rho_{i}(k)=\frac{1}{2 \pi}-\frac{1}{2 \pi} \sum_{\beta} \frac{\theta\left(2 \sin k_{j+1}-2 \Lambda_{\beta}\right)-\theta\left(2 \sin k_{j}-2 \Lambda_{\beta}\right)}{k_{j+1}-k_{j}} \sigma_{i}(\Lambda)\left|\Lambda_{\beta+1}-\Lambda_{\beta}\right|,
$$

where $k_{j} \leqslant k<k_{j+1}$ and $\Lambda_{\beta} \leqslant \Lambda<\Lambda_{\beta+1}$. We can fix $k$ now and consider (5.5) as an equation for $\rho_{i}(k)$.

To show the existence of a pointwise limit, we just need to show that the right side of (5.5) converges for all $k$. We know that the fraction on the right side converges to the derivative of $\theta$, if $\left|k_{j+1}-k_{j}\right| \rightarrow 0$. Since we did not prove that this happens for every $k$, let us divide the domain of $\rho$ into $Q \in[-\pi, \pi]$, defined by $Q=\left\{k \in[-\pi, \pi]:\left|k_{j+1}-k_{j}\right| \rightarrow 0\right\}$, and its complimentary $Q^{c}$.

In $Q^{c}$, the distance between the surrounding $k_{j}$ does not converge to zero. For a $k$ in the interior of $Q^{c}$, we can find an $\epsilon$ and a subsequence such that $\left|k_{j}-k\right|>\epsilon / 2$, for all $j$. That implies that the weak limit vanishes in this interval of lenght $\epsilon$ around $k$, since the integral of the characteristic function of any subset of this interval times $\rho_{i}$ converges to zero. Also, the subsequence will have a pointwise limit on this set that is equal to zero (almost everywhere). Since the boundary of $Q^{c}$ has measure zero, the value of the function at those points is not relevant in the context of $L^{p}$-spaces, which are defined by equivalence classes of functions that differ only on a set of measure zero.

Therefore, the subsequence $\left\{\rho_{i}\right\}$ has a pointwise limit in $Q^{c}$ that agrees with the weak limit. We will show that the same holds true in $Q$. A similar analysis can be done for the sequence $\left\{\sigma_{i}\right\}$. We define the set $B \in \mathbb{R}$ by $B=\left\{\Lambda \in \mathbb{R}:\left|\Lambda_{\alpha+1}-\Lambda_{\alpha}\right| \rightarrow 0\right\}$. In its 
complimentary $B^{c}$, the subsequence has a zero pointwise limit that agrees with the weak limit.

Now if $k \in Q$, the last term of (5.5) is

$$
\frac{\cos k}{\pi} \sum_{\beta}\left[\theta^{\prime}\left(2 \sin k-2 \Lambda_{\beta}\right)+E_{i}^{(1)}\left(k, \Lambda_{\beta}\right)\right] \sigma_{i}(\Lambda)\left|\Lambda_{\beta+1}-\Lambda_{\beta}\right|,
$$

where $E_{i}^{(1)}\left(k, \Lambda_{\beta}\right)$ is the error in approximating the ratio in (5.5) by the derivative of $\theta$. Since $\theta^{\prime \prime}$ is bounded, we have

$$
\sum_{\beta} E_{i}^{(1)}\left(k, \Lambda_{\beta}\right) \sigma_{i}(\Lambda)\left|\Lambda_{\beta+1}-\Lambda_{\beta}\right| \leqslant \sum_{\beta} \frac{C\left|k_{j+1}-k_{j}\right|}{N_{a}}=C m\left|k_{j+1}-k_{j}\right| \longrightarrow 0,
$$

as $i \rightarrow \infty$.

We need to show now that (5.6) converges to an integral. We first notice that

$$
\begin{array}{r}
\sum_{\beta} \theta^{\prime}\left(2 \sin k-2 \Lambda_{\beta}\right) \sigma_{i}(\Lambda)\left|\Lambda_{\beta+1}-\Lambda_{\beta}\right|=\int \theta^{\prime}\left(2 \sin k-2 \Lambda_{\beta}\right) \sigma_{i}(\Lambda) d \Lambda+ \\
+\int\left[\theta_{t r}^{\prime}\left(2 \sin k-2 \Lambda_{\beta}\right)-\theta^{\prime}\left(2 \sin k-2 \Lambda_{\beta}\right)\right] \sigma_{i}(\Lambda) d \Lambda,
\end{array}
$$

where $\theta_{t r}^{\prime}$ is the truncated version of $\theta^{\prime}$, defined by a piecewise constant function, whose value on the interval $\left[\Lambda_{\beta}, \Lambda_{\beta+1}\right)$ is equal to $\theta^{\prime}\left(2 \sin k-2 \Lambda_{\beta}\right)$. Again, since the second derivative of $\theta$ is bounded uniformly by a function in $L^{1}$, we can write

$$
\left|\theta_{t r}^{\prime}\left(2 \sin k-2 \Lambda_{\beta}\right)-\theta^{\prime}\left(2 \sin k-2 \Lambda_{\beta}\right)\right| \leqslant g(\Lambda)\left|\Lambda_{\beta+1}-\Lambda_{\beta}\right|,
$$

where $g(\Lambda) \in L^{1}(\mathbb{R})$. It follows that

$$
\lim _{i \rightarrow \infty} \int\left|\theta_{t r}^{\prime}\left(2 \sin k-2 \Lambda_{\beta}\right)-\theta^{\prime}\left(2 \sin k-2 \Lambda_{\beta}\right)\right| \sigma_{i}(\Lambda) d \Lambda \leqslant \lim _{i \rightarrow \infty} \int \frac{g(\Lambda)}{N_{a}} d \Lambda=0 .
$$

Since $\left\{\sigma_{i}\right\}$ has a weak limit, the first integral on the right side of (15.7) converges, and we have

$$
\lim _{i \rightarrow \infty} \rho_{i}(k)=\frac{1}{2 \pi}-\frac{\cos k}{\pi} \int_{B} \theta^{\prime}(2 \sin k-2 \Lambda) \sigma_{w}(\Lambda) d \Lambda,
$$

for all $k \in Q$. A similar analysis for $\sigma_{i}(\Lambda)$ yields

$$
\lim _{i \rightarrow \infty} \sigma_{i}(\Lambda)=-\frac{1}{\pi} \int_{B} \theta^{\prime}(2 \sin k-2 \Lambda) \rho_{w}(\Lambda) d \Lambda+\frac{1}{2 \pi} \int_{B} \theta^{\prime}\left(\Lambda-\Lambda^{\prime}\right) \sigma_{w}(\Lambda) d \Lambda,
$$

for all $\Lambda \in B$.

We just showed that the subsequences have a pointwise limit. But since $\left\{\rho_{i}\right\},\left\{\sigma_{i}\right\}$ are uniformly bounded, dominated convergence implied the existence of a strong limit, which equals the pointwise limit and the weak limit almost everywhere. Therefore, as we go to the thermodynamic limit on this subsequence, our $k$-s and $\Lambda$-s converge to distributions satisfying the integral equations

$$
\begin{aligned}
\rho(k) & =\frac{1}{2 \pi}+\cos k \int_{B} K(\sin k-\Lambda) \sigma(\Lambda) d \Lambda \\
\sigma(\Lambda) & =\int_{Q} K(\sin k-\Lambda) \rho(k) d k-\int_{B} K^{2}\left(\Lambda-\Lambda^{\prime}\right) \sigma(\Lambda) d \Lambda
\end{aligned}
$$


where we define

$$
\begin{aligned}
K\left(\Lambda-\Lambda^{\prime}\right) & =-\frac{1}{\pi} \theta^{\prime}\left(2 \Lambda-2 \Lambda^{\prime}\right), \\
K^{2}\left(\Lambda-\Lambda^{\prime}\right) & =-\frac{1}{2 \pi} \theta^{\prime}\left(\Lambda-\Lambda^{\prime}\right)=\int_{-\infty}^{\infty} K(\Lambda-x) K\left(x-\Lambda^{\prime}\right) d x .
\end{aligned}
$$

It was shown in LW2 that it is convenient to restrict the integral in $k$ to a domain where the function $\sin k$ is monotonic. Hence, we divide the range of integration $Q$ into $Q_{-}=Q \cap[-\pi / 2, \pi / 2]$ and $Q_{+}=Q \cap([-\pi,-\pi / 2) \cup(\pi / 2, \pi])$. The first thing to show is that for every $k=(\pi / 2+x) \in Q_{+}$, there is a $k=(\pi / 2-x) \in Q_{-}$.

Since the derivative of $f_{i}(k)$, as defined in (5.3) satisfies $f_{i}^{\prime}(k) \geqslant N_{a}$ for $k \in[-\pi / 2, \pi / 2]$, the set $Q_{-}$consists of a single interval $\left[-k^{*}, k^{*}\right]$ (possibly $[-\pi / 2, \pi / 2]$ ). Since $f(k) \geqslant$ $f\left(k^{*}\right)+N_{a}\left(k-k^{*}\right)$ for any $k \in\left[k^{*}, \pi-k^{*}\right]$, and the $k_{j}$-s are ordered, we conclude that the existence of a gap in the interval $\left[k^{*}, \pi / 2\right]$ implies that $Q_{+} \cap\left[\pi / 2, \pi-k^{*}\right]=\emptyset$, and for every positive $k$ in $Q_{+}$there is a corresponding point in $Q_{-}$given by the reflection with respect to $k=\pi / 2$. The same holds for for every negative $k$ in $Q_{+}$, where now we need to take the reflection with respect to $-\pi / 2$.

Therefore, if we define the set $Q_{+}^{r}$ by the reflection of $Q_{+}$with respect to $\pm \pi / 2$,

$$
Q_{+}^{r}=\left\{ \pm(\pi-k), k \in Q_{+} \cap[\pi / 2, \pi]\right\},
$$

and we will have $Q_{+}^{r} \subset Q_{-}$. Finally, we can avoid the integration in $Q_{+}$by noting that $K(\sin k-\Lambda)$ is an even function of $k-\pi / 2$, which implies by (5.10) that $\rho(k)-1 / 2 \pi$ is odd as a function of $k-\pi / 2$. Therefore, we can rewrite part of the first integral in (5.11) as

$$
\int_{Q_{+}}+\int_{Q_{+}^{r}} K(\sin k-\Lambda) \rho(k) d k=\int_{Q_{+}}+\int_{Q_{+}^{r}} \frac{K(\sin k-\Lambda)}{2 \pi}=\frac{2}{2 \pi} \int_{Q_{+}^{r}} K(\sin k-\Lambda)
$$

Now $\sin k$ is monotonic in the domain of the integral in $k$, and we can make the change in variables

$$
\begin{aligned}
t(x) & =\frac{\left(1-x^{2}\right)^{-1 / 2}}{2 \pi}, \\
f(x) & =\left(1-x^{2}\right)^{-1 / 2} \rho\left(\sin ^{-1} x\right),
\end{aligned}
$$

for $-1 \leqslant x \leqslant 1$. If $A, D$ are the images of $Q_{-}, Q_{+}^{r}$ under $\sin k$, the integral equations (5.10), (5.11) can now be written

$$
\begin{aligned}
f(x)= & t(x)+\int_{-\infty}^{\infty} K\left(x-x^{\prime}\right) B\left(x^{\prime}\right) \sigma\left(x^{\prime}\right) d x^{\prime} \quad, \quad x \in A, \\
\sigma(x)= & \int_{-\infty}^{\infty} K\left(x-x^{\prime}\right)\left(A\left(x^{\prime}\right) f\left(x^{\prime}\right)+D\left(x^{\prime}\right) t\left(x^{\prime}\right)\right) d x^{\prime} \\
& -\int_{-\infty}^{\infty} K^{2}\left(x-x^{\prime}\right) B\left(x^{\prime}\right) \sigma\left(x^{\prime}\right) d x^{\prime} \quad, \quad x \in B,
\end{aligned}
$$

where $A(x)=\chi_{A \backslash D}, B(x)=\chi_{B}$, and $D(x)=2 \chi_{D}\left(\chi_{X}\right.$ denotes the characteristic function of the set $X)$.

These equations have to be solved for $x$ in the sets defined above. However, we can let the right side of (5.12) and (5.13) define $f(x)$ and $\sigma(x)$ for any $x \in \mathbb{R}$, by setting $t(x) \equiv 0$ for $|x|>1$. Since the integrals only depend on $f(x)$ and $\sigma(x)$ at the original intervals, there is a one to one correpondence between the solutions to the extended equations and 
the solutions of the original equations. In particular, the new equations will have a unique solution if and only if the solution to (5.12) and (5.13) is unique.

It is convenient to write the integral equations in the operator form

$$
\begin{aligned}
f & =t+\hat{K} \hat{B} \sigma, \\
\sigma & =\hat{K} \hat{A} f+\hat{K} \hat{D} t-\hat{K}^{2} \hat{B} \sigma,
\end{aligned}
$$

where $\hat{K}$ is the convolution with $K$ and $\hat{A}, \hat{B}, \hat{D}$ are the multiplication by $A, B$ and $D$.

The proof that (5.14) and (5.15) have a unique solution for given sets $Q$ and $B$ was done by Lieb and $\mathrm{Wu}$ in $[\mathrm{LW} 2$. We did not prove here that the sets $Q$ and $B$ are uniquely defined for every $n=N / N_{a}$ and $m=M / N_{a}$, although we believe they are (and we also belive that they are given by intervals $\left.Q=\left[-k_{\max }, k_{\max }\right], B=\left[-\Lambda_{\max }, \Lambda_{\max }\right]\right)$. However, this is not a problem for the absolute ground state of the half filled band, in which case an explicit solution for (5.14) and (5.15) can be found. In this case, we have $n=1$ and $m=1 / 2$, and Lieb and Wu proved that $Q=[-\pi, \pi]$ and $B=\mathbb{R}$. Let us restrict now to this case.

Since the solution is unique, and every subsequence of $\left\{\rho_{i}\right\},\left\{\sigma_{i}\right\}$ contains a further subsequence that converges to the same $\rho$ and $\sigma$, we can actually prove that the whole sequence converges. Otherwise, we would be able to find a subsequence such that $\| \rho_{n_{i}}-$ $\rho \|>\epsilon$ or $\left\|\sigma_{n_{i}}-\sigma\right\|>\epsilon$, which is a contradiction.

The limit distribution in the thermodynamic limit for $k$-s and $\Lambda$-s are then given by the solution of (5.10), (5.11), obtained in [LW1],

$$
\begin{aligned}
\rho(k) & =\frac{1}{2 \pi}+\frac{\cos k}{\pi} \int_{0}^{\infty} \frac{\cos (\omega \sin k) J_{0}(\omega)}{1+e^{\omega U / 2}} d \omega, \\
\sigma(\Lambda) & =\frac{1}{2 \pi} \int_{0}^{\infty} \frac{J_{0}(\omega) \cos (\omega \Lambda)}{\cosh (\omega U / 4)} d \omega
\end{aligned}
$$

which yield the ground state energy

$$
E=-2 N_{a} \int_{-\pi}^{\pi} \rho(k) \cos k d k=-4 N_{a} \int_{0}^{\infty} \frac{J_{0}(\omega) J_{1}(\omega)}{\omega\left(1+e^{\omega U / 2}\right)} d \omega
$$

where $J_{n}$ is the Bessel function of order $n$.

\section{Conclusions}

We have concluded the proof that the Bethe Ansatz equations, and in particular the Lieb-Wu equations (1.5) and (1.6), have a solution for finite $M, N$ and $N_{a}$. The solution is indeed real and ordered in the indices $j$ and $\alpha$, as assumed by Lieb and $\mathrm{Wu}$ in the derivation of the integral equations for the model in the thermodynamic limit.

We have also shown the existence of a continuous set of solutions extending from any $U>0$ to $U=\infty$. The continuity is important to show that the state obtained by the Lieb-Wu equations is indeed the ground state of the system, provided it does not vanish. Since we know that the state obtained is the genuine ground state of the system at $U=\infty$, and that the ground state is nondegenerate for any $U>0$ (for $M, M^{\prime}$ odd), a normalizable state given by the Bethe Ansatz should also give us the ground state of the system for any $U$, since the energy cannot discontinuously jump from an excited level to the ground state as we increase the interaction strength from a finite value to the limit $U=\infty$.

To conclude the proof for a finite lattice, we show that the norm of the wavefunction obtained by the Bethe Ansatz equations is well defined and not zero, for all $U>0$, except for finitely many values of $U$. If $U_{0}$ is one of this values, the correct eigenfunction will be 
given by the limit $U \rightarrow U_{0}$ of the normalized wavefunction, since $U_{0}$ is an isolated zero or singularity.

Going from the finite lattice to the thermodynamic limit, we focus primarily on the half-filled band $N=N_{a}$. In this case, the set of $k$-s converge to a distribution $\rho(k)$ on the interval $[-\pi, \pi]$. If we consider the absolute ground state with $M=M^{\prime}$, the set of $\Lambda$-s converge to a distribution $\sigma(\Lambda)$ in $\mathbb{R}$, and we showed that $\rho(k)$ and $\sigma(\Lambda)$ satisfy the integral equations derived by Lieb and $\mathrm{Wu}$, whose solution is given by (5.16), (5.17). For other fillings, we also prove the convergence (at least for a subsequence) of the distribution to the solution of the integral equations, but we do not prove uniqueness of the sets $Q$ and $B$ that contain the support of $\rho$ and $\sigma$.

The author is indebted to Elliott H. Lieb for useful comments and suggestions.

\section{Appendix A. Groebner bases}

Every ideal $I\left(f_{1}, \ldots, f_{m}\right)$ generated by the polynomials $\left\{f_{k}\right\}_{k=1}^{m} \neq\{0\}$ has a Groebner basis. The general idea in constructing a Groebner basis is to eliminate one by one the variables of the polynomials $f_{k}$, obtaining a set of polynomials $G=\left\{g_{k}\right\}_{k=1}^{l}$ that generate the same ideal as $\left\{f_{k}\right\}_{k=1}^{N}$. One of its main applications is to solve systems of polynomial equations, since reducing the number of variables simplifies the task of finding solutions. This process can be thought of as a generalization of the process of solving a system of linear equations by elimination of variables. We will present here the essential facts about Groebner basis in order to understand the results of section 4. The interested reader should refer to CLO for a precise definition of a Groebner basis and the algorithm used to obtain it.

Given an ideal $I \subset \mathbb{C}\left[x_{1}, \ldots, x_{n}\right]$, we can define the $k$-elimination ideal by

$$
I_{k}=I \cap \mathbb{C}\left[x_{k+1}, \ldots, x_{n}\right] .
$$

$I_{k}$ is the set of all polynomials in $I$ that do not depend on the first $k$ variables $x_{k}$. What makes the Groebner basis special is that

$$
G_{k}=G \cap \mathbb{C}\left[x_{k+1}, \ldots, x_{n}\right]
$$

is also a basis for $I_{k}$.

As a consequence of this property, if the variety defined by (4.2) consists of finitely many points, $G_{n-1} \neq\{0\}$, and we can solve the corresponding equations for $x_{n}$, to find $V\left(I_{n-1}\right)$. Then, we can move on to $G_{n-2}$, and solve it for $x_{n-2}$, finding $V\left(I_{n-2}\right)$, and so on. In the end, we will find $V(I)$ just by solving polynomial equations in one variable. We are ordering the variables here by $x_{1}>x_{2}>\cdots>x_{n}$. This ordering determines which variables will be eliminated first. Of course we can choose any other ordering, and we will obtain a different Groebner basis, but the set of solutions should be the same

As one example, consider the set of polynomial equations $f_{1}=x^{2}+y^{2}+z-1=0$, $f_{2}=x+y+z-1=0$ and $f_{3}=x+y-z^{2}-1=0$. Fixing the ordering $x>y>z$, the ideal $I\left(f_{1}, f_{2}, f_{3}\right)$ has $G=\left\{x+y+z-1, y^{2}-y+y z-z, z^{2}+z\right\}$ as a possible Groebner basis. Therefore, the solution to the original equations will satisfy

$$
\begin{array}{r}
x+y+z-1=0, \\
y^{2}-y+y z-z=0, \\
z^{2}+z=0,
\end{array}
$$

which can be easily solved to get $V(I)=\{(0,1,0),(1,0,0),(1,1,-1)\}$. 
Clearly our solutions $V(I)$ could be more complicated than isolated points. It could contain subsets of higher dimension. In this case, we would have a slightly different basis. Consider for instance the set of solutions of $f_{1}=f_{2}=0$. It clearly has infinitely many solutions, and $G=\left\{x+y+z-1,2 y^{2}-2 y+2 y z+z^{2}-z\right\}$ is a Groebner basis. We see that $G_{2}=\{0\}$ and $V\left(I_{2}\right)=\mathbb{C}$. In this case, we could not eliminate $x$ and $y$, since we have solutions for infinitely many values of $z$. In summary, the number of variables we can actually eliminate by calculating the Groebner basis depends on the dimension of $V(I)$.

\section{REFERENCES}

[Be] H. A. Bethe, Zur Theorie der Metalle: I. Eigenwerte und Eigenfunktionen der linearen Atom Kette, Zeits. f. Physik 71, 205-226 (1931)

[CLO] D. Cox, J. Little and D. O'Shea, Ideals, Varieties, and Algorithms, Springer-Verlag, New York, USA, 1992

[G] M. Gaudin, Travaux de Michel Gaudin: Modèles exactement résolus, Les Éditions de Physique, Paris \& Cambridge, USA, 1995

[GP] V. Guillemin and A. Pollack, Differential Topology, Prentice-Hall Inc., Englewood Cliffs, NJ, USA, 1974

[GS] D. H. Gottlieb and G. Samaranayake, The index of discontinuous vector fields, New York J. Math. 1, 130-148 (1995)

[H] J. Hubbard, Electron correlation in narrow energy bands, Proc. Roy. Soc. (London) A 276, 238-257 (1963)

[LL] E. H. Lieb and M. Loss Analysis, 2nd edition, Amer. Math. Soc., 2001.

[LW1] E. H. Lieb and F. Y. Wu, Absence of Mott transition in an exact solution of the short-range one-band model in one dimension, Phys. Rev. Lett. 20, 1445-1448 (1968)

[LW2] E. H. Lieb and F. Y. Wu, The one-dimensional Hubbard model: A reminiscence, Physica A 321, 1-27 (2003)

[W] F. Woynarovich, Excitations with complex wavenumbers in a Hubbard chain: I. States with one pair of complex wavenumbers, J. Phys. C 15, 85-96 (1982)

[Y] C. N. Yang Some exact results for the many-body problem in one dimension with repulsive deltafunction interaction, Phys. Rev. Lett. 19, 1312-1314 (1967)

[YY] C. N. Yang and C. P. Yang One-dimensional chain of anisotropic spin-spin interactions. I. Proof of Bethe's hypothesis for ground state in a finite system, Phys. Rev. 150, 321-327 (1966) 